\title{
Review Article \\ Zebrafish as a Model for the Study of Human Myeloid Malignancies
}

\author{
Jeng-Wei Lu, ${ }^{1}$ Meng-Shan Hsieh, ${ }^{1}$ Heng-An Liao, ${ }^{1}$ Yi-Ju Yang, \\ Yi-Jung $\mathrm{Ho}^{2,3}$ and Liang-In Lin ${ }^{1,4}$ \\ ${ }^{1}$ Department of Clinical Laboratory Sciences and Medical Biotechnology, College of Medicine, \\ National Taiwan University, No. 1 Chang-Te Street, Taipei 100, Taiwan \\ ${ }^{2}$ Institute of Preventive Medicine, National Defense Medical Center, No. 172 Dapu Road, New Taipei City 237, Taiwan \\ ${ }^{3}$ Graduate Institute of Life Sciences, National Defense Medical Center, No. 161, Section 6, Minquan East Road, Taipei 114, Taiwan \\ ${ }^{4}$ Department of Laboratory Medicine, National Taiwan University Hospital, No. 7 Chung-Shan Southern Road, Taipei 100, Taiwan
}

Correspondence should be addressed to Liang-In Lin; lilin@ntu.edu.tw

Received 26 September 2014; Revised 11 December 2014; Accepted 15 December 2014

Academic Editor: Monica Fedele

Copyright (C) 2015 Jeng-Wei Lu et al. This is an open access article distributed under the Creative Commons Attribution License, which permits unrestricted use, distribution, and reproduction in any medium, provided the original work is properly cited.

Myeloid malignancies are heterogeneous disorders characterized by uncontrolled proliferation or/and blockage of differentiation of myeloid progenitor cells. Although a substantial number of gene alterations have been identified, the mechanism by which these abnormalities interact has yet to be elucidated. Over the past decades, zebrafish have become an important model organism, especially in biomedical research. Several zebrafish models have been developed to recapitulate the characteristics of specific myeloid malignancies that provide novel insight into the pathogenesis of these diseases and allow the evaluation of novel small molecule drugs. This report will focus on illustrative examples of applications of zebrafish models, including transgenesis, zebrafish xenograft models, and cell transplantation approaches, to the study of human myeloid malignancies.

\section{Introduction}

Myeloid malignancies, including myeloproliferative neoplasms (MPNs), myelodysplastic syndrome (MDS), and acute myeloid leukemia (AML), are heterogeneous disorders characterized by uncontrolled proliferation or/and blockage of differentiation of abnormal myeloid progenitor cells [1]. MPNs are clonal hematopoietic stem cell disorders characterized by proliferation of one or more of the myeloid lineages. MDS is also a clonal hematopoietic disorder characterized by the simultaneous proliferation and apoptosis of hematopoietic cells, which leads to a normal state or hypercellularity of the bone marrow $(\mathrm{BM})$ and pancytopenia in the peripheral blood (PB).

A substantial number of studies have revealed that MPN or MDS can evolve into AML in some patients [2]. AML is a heterogeneous disease that results from the clonal expansion of myeloblasts in the $\mathrm{BM}$ and $\mathrm{PB}$ and may involve only one or all myeloid cell lineages $[3,4]$. Numerous recurrent gene fusions (such as $\mathrm{t}(15 ; 17) / P M L-R A R A, \mathrm{t}(8: 21) / A M L 1-$ $E T O$, inv(16)/CBFB-MYH11, and $\mathrm{t}(9 ; 11) / M L L-M L L T 3)$ and point mutations (such as NPM1, FLT3, KIT, and CEBPA), associated with class I (proliferation advantage) and class II (differentiation blockage) mutations, have been identified over the past several decades. Recent comprehensive studies with whole-genome sequencing or whole-exome sequencing, RNA and microRNA sequencing, and DNA methylation analysis have successfully classified the related genes into nine categories based on the function of the mutated genes, including transcription factor gene fusions, NPM1, tumor suppressor genes, genes with chromosome modifiers, genes with DNA methylation, activated signaling genes, myeloid transcription factor genes, cohesioncomplex genes, and spliceosome-complex genes [5]. However, the mechanisms which by these identified alterations interact to induce AML and distinguish driver from passenger mutations in leukemogenesis have yet to be elucidated. 
The zebrafish is a popular research model in biomedical research fields, including embryonic development, human diseases, cancer studies, toxicity, and chemical screening [6]. From a genetic point of view, the current zebrafish genome has been fully sequenced, and many genes are conserved between the human and zebrafish genomes. The zebrafish genome is composed of 25 chromosomes and essentially contains the full vertebrate repertoire of genes. More importantly, approximately $84 \%$ of the human genes that cause diseases have a zebrafish ortholog [7]. Although zebrafish and mammalian hematopoietic organs belong to different sites, the genetic and cellular levels of hematopoiesis are conserved between these groups. Zebrafish carry their hematopoietic stem cells in the kidney marrow and have blood cell types similar to human beings $[8,9]$.

Compared to the mouse model, the zebrafish model is very suitable for large-scale genetic and high-throughput screening in many ways, and it allows more powerful induction of tumors by carcinogens [10]. The first zebrafish model of hematological malignancy was generated in 2003 using the mouse $M y c$ gene driven by recombinase activating gene 2 (rag2) promoters. This zebrafish model faithfully developed T-cell lymphoblastic leukaemia (T-ALL) that closely parallels the human disorder subtype [11]. Recently, the zebrafish system has been used to study genetic pathways and understand the pathogenesis involved in human cancers [12-14]. Thus, the zebrafish provides a unique model system to study disease mechanisms in vivo. This paper summarizes the benefits of using the zebrafish model to study myeloid leukaemogenesis, reviews current zebrafish models of specific myeloid malignancies, and gives future directions for zebrafish models in the study of human cancer. In addition, the bridge between basic science and translational research will be discussed.

\section{Overview of Zebrafish Hematopoiesis}

Hematopoiesis is a complex process that utilizes many transcription factors to form all of the blood cell lineages from common multipotent hematopoietic stem cells (HSCs). Various experimental tools and methods have been established to facilitate the understanding of hematopoiesis and blood-related disease mechanisms. The zebrafish is an ideal animal model to study hematopoietic development due to its experimental advantages. Although they possess some characteristics that are different from other vertebrates and mammals, such as the site of hematopoiesis, the lineages of blood cells as well as the transcriptional regulators associated with the fate of blood cells have been evolutionarily conserved $[15,16]$. Similar to other vertebrates, two major waves of hematopoiesis, the primitive and definitive waves, sequentially occur in zebrafish hematopoiesis [17]. The first wave of definitive hematopoiesis produces a transient population of cells, termed erythroid myeloid progenitors (EMPs), in the posterior blood island (PBI). The population of primitive myeloid cells is predominantly different from that of hemangioblasts at the anterior lateral mesoderm (ALM) between 12 and 24 hours after fertilization (hpf) [18, 19]. However, the posterior lateral mesoderm (PLM) is the major location of primitive erythroid progenitors and some myeloid cells [20-23]. Beginning at $24 \mathrm{hpf}$, these primitive blood cells enter the circulation and are distributed throughout the embryo. The definitive wave of hematopoiesis begins at approximately $26-30 \mathrm{hpf}$, and multipotent HSCs emerge from the hemogenic endothelium that resides in the ventral aspect of the dorsal aorta in the aorta-gonad-mesonephros (AGM) region $[21,24,25]$. They then migrate to the posterior region of the tail-caudal hematopoietic tissue (CHT), along with the circulation, after $36 \mathrm{hpf}$, and differentiate into cells during that period of time $[26,27]$. Ultimately, HSCs from the AGM and CHT seed the kidney marrow approximately 4 days after fertilization (dpf) and give rise to all lineages of blood cells for the remainder of adult life. The CHT is analogous to the mammalian fetal liver or placenta, while the kidney marrow is functionally equivalent to mammalian bone marrow. In addition, some of HSCs also seed the thymus, where lymphopoiesis is initiated at approximately $3 \mathrm{dpf}$, and remain permanently after the maturation of lymphocytes.

Several zebrafish transcription factors that regulate hematopoiesis have been identified. The early stage markers gata2, lmo2, fli1, and scl (stem cell leukemia) are master regulators that are coexpressed in both the ALM and PLM, where hemangioblast development occurs, from the 2nd to the $3 \mathrm{rd}$ somite stages $[20,21,23]$. These genes are expressed in the PLM and later in the intermediate cell mass (ICM) [2831]. $l \mathrm{mo} 2, \mathrm{flil}$, and $\mathrm{scl}$ may function as crucial factors necessary for normal erythroid and myeloid development [30-32]. In addition, gatal and spil (pu.1) are also involved in primitive hematopoiesis. Gatal, a transcription factor essential for erythropoiesis, is first detectable in cells of the PLM at $12 \mathrm{hpf}$ and then in the anterior ICM $[33,34]$. Conversely, expression of spil, an ETS transcription factor required for myeloid cell development, appears in the ALM and ICM between 16 and $30 \mathrm{hpf}$ [35]. Later, these spil expressing progenitor cells differentiate into macrophages and granulocytes that express l-plastin (lcp1) and myeloperoxidase (mpo), respectively $[18,36]$. Runxl has been shown to be required for definitive hematopoiesis based on knockdown experiments that resulted in reduced c-myb expression and lymphopoiesis, but it produces no apparent effect on the development of primitive hematopoiesis [24, 37-39].

\section{Transgenic Technology for Studying Myeloid Malignancies Using Zebrafish}

The available zebrafish transgenic technology has improved over the last two decades [40, 41]. Different systems have been used in transgenic zebrafish models, such as injection of linear DNA [40] or supercoiled plasmid DNA [41, 42] or injection of recombinant bacterial artificial chromosomes into embryos [42]. In recent years, a new transgenic technology related to Tol2-mediated transgenesis has been established. The Tol2 element is a naturally occurring active transposable element found in vertebrate genomes. The Tol2 transposon system is considered a useful gene transfer vector in organisms ranging from fish to mammals [43]. Tol2mediated transgenesis is an excellent method for creating 


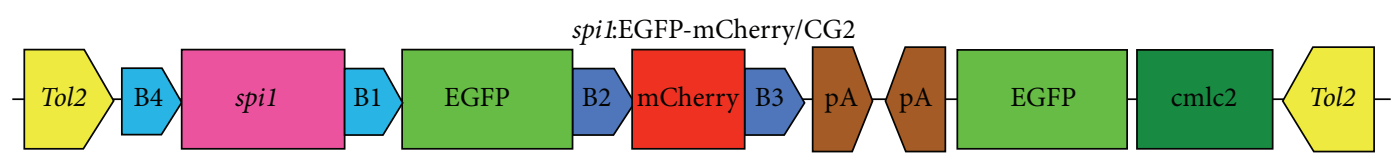

(a)
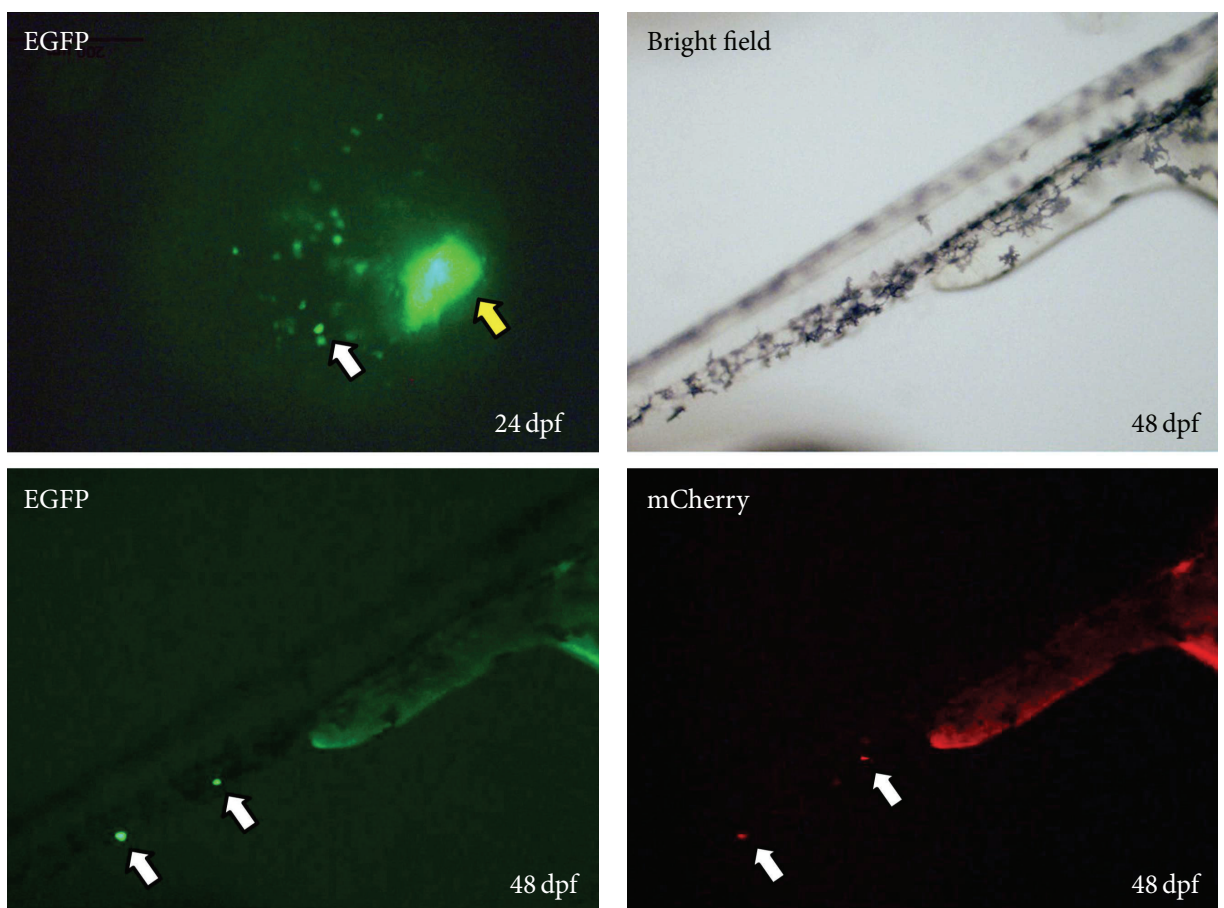

(b)

FIGURE 1: Expression of the spil:EGFP-mCherry/CG2 transgenic fish. (a) Diagram of the spil:EGFP-mCherry/CG2 construct that contains Tol2 sequences and the cmlc2:GFP expression cassette. (b) Fluorescent images of TG (spil:EGFP-mCherry/CG2) embryos at 24 or 48 hpf. The white arrowhead indicates spil expressing myeloid cells and the yellow arrowhead fluorescence in heart.

transgenic zebrafish using coinjection of Tol2 RNA, and the DNA fragment surrounded by the Tol2 element transposon can be efficiently excised and integrated into the zebrafish genome $[44,45]$.

Constitutive expression systems have proven useful, but it is often observed that the expression of oncogenes may cause serious tumors and early lethality, preventing the complete characterization of their effects. Therefore, the use of an induction system can help to solve this problem. For example, Tet-On, Tet-Off, Mifepristone, Cre-loxP, Heat-shock, and GAL4-UAS inducible systems can be used. The duration and dosage of oncogene expression can be monitored with such a system, thus allowing the spatiotemporal control of gene expression [46].

A green fluorescent protein (GFP) reporter can be a useful marker for determining if mammalian promoters and ubiquitous or endogenous tissue-specific promoters can drive downstream transgene expressions in zebrafish [43]. It has been noted that spil plays an important role in the development of myeloid (granulocytes and monocytes/macrophages) cells in zebrafish, and the spil promoter can drive myeloidspecific expression in zebrafish. Ward et al. [47] and Hsu et al. [48] identified a $5.3 \mathrm{~kb}$ and a $9.0 \mathrm{~kb}$ promoter fragment of zebrafish sequences upstream of the spil coding sequence that is sufficient to drive the expression of downstream genes. Recently, a transgenic fish line with a spil:EGFPmCherry/CG2 construct was established for studying the cooperation of various genetic aberrations found in myeloid malignancies (Figure 1), and this model may become a useful zebrafish model for exploring leukemogenesis in myeloid cells.

\section{Myeloid Malignancies Animal Models in Zebrafish}

RAS mutations associated with cancer frequently occur in patients with AML, suggesting a functional role for Ras in leukemogenesis. AML1/ETO rearrangements are detected frequently in AML, especially M2, and are associated with a relatively good prognosis [49]. K-RASG12D interacts with $A M L 1 / E T O$ to induce acute monoblastic leukemia in a mouse model [50]. The NUP98-HOXA9 fusion oncogene is related to an inferior prognosis in de novo and treatment-related AML and induces AML in mouse models [51]. NUP98HOXA9 is associated with increased cell proliferation and survival as well as drug metabolism [52]. The MOZ/TIF2 
fusion oncogene was described in a specific subgroup of AML that represents approximately 5\% of M4/M5 AML [53]. The transforming properties of MOZ/TIF2 have been demonstrated in mouse committed myeloid progenitors in vitro and in vivo $[54,55]$. Constitutive activation of Stat5 has been observed in hematological malignancies and is often triggered by leukemic oncoproteins, such as Tel-Jak2 and Bcr$A b l$, and Stat 5 has been shown to be involved in mediating the leukemic effects [56, 57]. Amplification of MYCN (N$M y c$ ) is frequently found in AML patients and is considered a well-established poor prognostic marker in this disease. Overexpression of MYCN rapidly causes AML in mouse models $[58,59]$. In AML patients, FLT3 and NPM have important prognostic implications on the treatment outcome. Patients with mutated NPM have a favorable outcome, while patients with mutations in the FLT3 gene generally have a poor prognosis. NPM1 and FLT3-ITD mutations interact to induce AML in mouse models [60].

Several zebrafish models associated with myeloid malignancies have been reported. In a transgenic zebrafish model, $K-R A S G 12 D$ was expressed under the actin promoter and induced MPNs [61]. Induction of NUP98-HOXA9 fusion genes in transgenic zebrafish under the spil promoter leads to MPNs at 19-23 months of age. However, in contrast to the mouse models, none of the NUP98-HOXA9 transgenic fish developed AML [62-64]. Lewis et al. addressed the transient expression of constitutively active Stat5 (H298R/N714Fmutant) in zebrafish, which leads to increased numbers of early and late myeloid cells, erythrocytes, and B cells [65]. Bolli et al. found that transient expression of the NPM1 mutant in zebrafish increases the number of definitive hematopoietic cells, including erythromyeloid progenitors, in the posterior blood island and $c-m y b / c d 41+$ cells in the ventral wall of the aorta [66].

In a transgenic zebrafish model, the tel-jak2a fusion oncogene was expressed in embryos under the control of the spil promoter, which resulted in disruption of embryonic hematopoiesis, including anemia and expansion of the myeloid compartment [67]. Transient expression of AML1ETO under the control of a CMV promoter in zebrafish embryos caused disruption of normal hematopoiesis, aberrant circulation, internal hemorrhages, and cellular dysplasia [24]. Induction of AML1-ETO transgenic zebrafish with the hsp70 promoter led to reprogramming of the multipotent hematopoietic progenitor cells from the erythroid cells to the myeloid cells in primitive hematopoiesis and disruption of definitive hematopoiesis in embryonic zebrafish [68].

Transient expression of FLT3-ITD in zebrafish embryos induced ectopic myeloid cell expansion and clustering, which were ameliorated by AC220 and associated with stat5, erk1/2, and akt phosphorylation. Overexpression of the FLT3ITD/TKD (D835Y) double mutation in zebrafish embryos conferred resistance to AC220 treatment. This zebrafish model may be useful for assessing the pathogenic significance and therapeutic potential of novel gene mutations [69].

In a transgenic zebrafish model of AML, MYCN, under the control of a $C M V$ minimal promoter and a MOZ/TIF2 fusion gene generated by the inv(8)(p1lq13) chromosomal abnormality expressed under the spil promoter, resulted in altered hematopoiesis and was characterized by invasion of the kidney marrow by immature myeloid cells $[58,70]$. Until now, only two published zebrafish AML models have developed overt leukemia (Table 1).

Recently, a transgenic zebrafish model that expresses AML1-ETO oncogenes has been reported as an excellent animal model for uncovering new therapeutic targets involved in oncogene-regulated hematopoietic differentiation [71]. According to these studies, the use of human genes produces greater fidelity and reliability in the translation of results associated with the interaction of molecular signaling pathways and disease therapy. In conclusion, the zebrafish is an excellent animal model for elucidating the mechanisms of leukemogenesis and provides an effective drug screening platform.

\section{Drug Screening in Zebrafish Using Xenograft Model}

One of the most extensively used in vivo animal models in the investigation of molecular mechanisms is xenotransplantation in immune-deficient animals. The zebrafish genome is nearly $100 \%$ homologous to the human genome in key domains [72]. The xenograft animal model has been utilized to investigate tumor biology, especially tumor cell proliferation, invasion, metastasis, and angiogenesis for decades, and the zebrafish model has been used as an alternative to mammalian models to assess the efficacy and toxicity of cancer drugs since 2005 [73].

Engrafting of human tumor cells into the yolk sac of zebrafish embryos at $48 \mathrm{hpf}$ can be used as a xenograft model of zebrafish due to the lack of an adaptive immune response at this stage [74]. To achieve maximum embryo transparency, embryos were incubated in egg medium with $0.3 \%$ phenylthiourea (PTU) to prevent pigment formation. As in mouse systems, the in vivo spatial resolution of the adult mouse is limited due to the normal opacification of the skin and subdermal structures. However, the body of zebrafish embryos is transparent, which allows the observation of labeled tumor cells and evaluation of the effects of cancer drugs. Due to the size of zebrafish embryos, high-throughput drug screening can be conducted in a 96-well format [74]. Several reports have verified that the results from zebrafish xenograft models are similar to those from mouse models [75, 76]. Taken together, the findings suggest that the zebrafish model may be a rapid, simple, sensitive, and reproducible xenograft model compared to the mouse model (Table 2).

Leukemia cells labeled with CM-Dil, a lipophilic fluorescent tracking dye, were injected into 2-day-old zebrafish larvae and used to evaluate the efficacy of imatinib and all-trans retinoic acid (ATRA) on the proliferation of K562 cells and NB4 cells, respectively [6], and these cells have also been used in ex vivo cell proliferation assays [77]. A novel phenotype-based in vivo screening method that uses leukemia stem cells (LSCs) xenotransplanted into zebrafish has been demonstrated. Aldehyde dehydrogenase-positive $(\mathrm{ALDH}+)$ cells reflecting LSCs were implanted into young zebrafish at $48 \mathrm{hpf}$, and the efficacy of various therapeutic 
TABLE 1: Zebrafish animal models of myeloid malignancies.

\begin{tabular}{|c|c|c|c|}
\hline Gene name & Promoter and expression construct & Type & Refs \\
\hline MYCN & MYCN-HSE-EGFP & AML & [58] \\
\hline MYST3/NCOA2 & spi-1-MYST3/NCOA2-EGFP & AML & [70] \\
\hline kRASG12D & $\begin{array}{l}\text { B-actin-LoxP-EGFP-LoxP-kRASG12D; } \\
\text { hsp70-Cre }\end{array}$ & MPD & {$[61]$} \\
\hline NUP98-HOXA9 & $\begin{array}{l}\text { spi-1-loxP-EGFP-loxP-NUP98-HOXA9; } \\
\text { hsp70-Cre }\end{array}$ & MPN & {$[62]$} \\
\hline Stat5.1 mutants & Constitutively active mutants of Stat5.1 & $\begin{array}{l}\text { Tumor-like lesions; increased numbers of early and late } \\
\text { myeloid cells, erythrocytes, and B cells }\end{array}$ & {$[65]$} \\
\hline NPM1 mutant & pCS2cmv-NPM1c-EGFP & $\begin{array}{l}\text { No AML; increased numbers of definitive } \\
\text { hematopoietic cells }\end{array}$ & {$[66]$} \\
\hline tel-jak2a & pCS2cmv-Flag-tel-jak2a; spi-1-Flag-tel-jak2a & $\begin{array}{l}\text { No AML; anemic; perturbed intermediate cell mass; } \\
\text { accumulation of large cells near the heart }\end{array}$ & {$[67]$} \\
\hline$A M L 1$ & pCS2cmv-runxl & No AML; enlarged heart and ectopic blood & {$[24]$} \\
\hline AML1-ETO & pCS2cmv-RUNX1-CBF2T1 & $\begin{array}{l}\text { No AML; defective development of blood and } \\
\text { circulation and internal hemorrhaging }\end{array}$ & [24] \\
\hline AML1-ETO & hsp-AML1-ETO & $\begin{array}{l}\text { No AML; loss of gatal hematopoietic cells in the } \\
\text { posterior blood islands }\end{array}$ & {$[68]$} \\
\hline $\begin{array}{l}\text { AML1-ETO + Gro3 } \\
\text { MO }\end{array}$ & hsp-AML1-ETO & $\begin{array}{l}\text { No apparent AML; enhanced the accumulation of blast } \\
\text { cells }\end{array}$ & {$[68]$} \\
\hline \multirow{3}{*}{ FLT3-ITD } & CMV-FLT3-ITD-T2a-EGFP & Ectopic myeloid cell expansion & \multirow{3}{*}[69]{} \\
\hline & CMV-FLT3-TKD-T2a-EGFP & Ectopic myeloid cell expansion resistant to AC220 & \\
\hline & CMV-FLT3-ITD-TKD-T2a-EGFP & Conferred resistance to AC220 treatment & \\
\hline
\end{tabular}

AML: acute myeloid leukemia; MPD: myeloproliferative disorder; MPN: myeloproliferative neoplasm; MO: morpholino.

TABLE 2: The advantages and weaknesses in the xenograft model of zebrafish and mouse.

\begin{tabular}{|c|c|c|c|}
\hline & Item & Zebrafish & Mouse \\
\hline \multirow{8}{*}{ Strengths } & Maintenance cost & Low & Available \\
\hline & Maintenance space & Small & Large \\
\hline & Offspring & Large number & Less number \\
\hline & Immune system & Lack in early zebrafish embryos & Innate and adaption \\
\hline & Observation & $\begin{array}{l}\text { Visualization (transparency and transgenic } \\
\text { lines) }\end{array}$ & Limitation \\
\hline & Readout time & Hours to days & Days to months \\
\hline & $\begin{array}{l}\text { Cell numbers required for } \\
\text { xenotransplantation per animal }\end{array}$ & Less & Large \\
\hline & High-throughput drug screening & Available & Limitation \\
\hline \multirow{5}{*}{ Weaknesses } & Size of organs/vessels & Small & Large \\
\hline & Body temperature & Low & High \\
\hline & Lack of organs & Breast, lung, etc. & \\
\hline & Zebrafish antibodies & Limitation & More \\
\hline & Adult immune-permissive lines & Unavailable & Available \\
\hline
\end{tabular}

agents was evaluated by high-content imaging [78]. From the transplanted tumor clusters, the number of migrating tumors and their areas were measured within concentric rings at a defined distance from the main tumor by calculating the area, total luminance value, and average radius of the tumor [62]. For cell proliferation assays, positive embryos were divided into two groups; one group was maintained at $35^{\circ} \mathrm{C}$ for $24 \mathrm{~h}$, while the other group was incubated with or without drug for $72 \mathrm{~h}$. At the end of each time period, the embryos were enzymatically dissociated into a single cell suspension, and the number of fluorescent cells in the suspension was counted. The number of fluorescent cells present at $72 \mathrm{~h}$ divided by the number of fluorescent cells present at $24 \mathrm{~h}$ represents the fold increase in the cell number 
[78]. These studies established the efficacy of a zebrafish xenograft platform as a rapid assessment of the effect of novel compounds on leukemia cell proliferation in vivo. Taken together, these findings suggest that the zebrafish xenograft model can be used as a platform for drug screening, a tool to rapidly assess the efficacy of novel compounds on the proliferation of human leukemia cells ex vivo and for providing information for subsequent preclinical mouse studies and clinical trials.

\section{Zebrafish Models for Hematopoietic Cell Transplantation Biology}

Hematopoietic stem cells are the source of all the blood cells needed by an organism during its lifetime. The study of hematopoiesis has been markedly facilitated by hematopoietic stem cell transplantation (HSCT), which involves transplanting donor blood cell populations into recipient animals. It is known that immune-matching is not required in embryonic recipients less than 5 days after fertilization because thymic development does not occur until that time in zebrafish [79]. Therefore, HSCT could be applied in experiments related to the development of quantitative long-term repopulating assays and the generation of histocompatible zebrafish lines [80].

The first hematopoietic cell transplantation in zebrafish revealed that hematopoietic cell transplantation could rescue multilineage hematopoiesis in embryonic lethal $\mathrm{gatal}^{-1-}$ mutants over six months of age [15]. The 2-day-old embryos were used as transplant recipients to partly circumvent graft rejection by performing short-term multilineage hematopoietic engraftment [15]. Subsequently, Traver et al. demonstrated an allogeneic HSCT into adult recipient zebrafish conditioned by a sublethal dose of gamma irradiation and established transplantation assays to evaluate the function of HSCs in zebrafish [81]. Self-renewal is a feature of cancer, and Smith et al. initially demonstrated high-throughput imaging methods to experimentally assess cell transplantation and evaluate the gene pathways involved in cancer self-renewal [82]. However, these approaches require donor cells from the same strain of syngeneic zebrafish or the recipient immune system to be transiently ablated by whole-body $\gamma$ irradiation before transplantation [15, 81, 82], making longterm engraftment studies difficult. In 2011, lethally irradiated animals could be rescued by transplantation of whole kidney marrow cells. The key zebrafish genes at the major histocompatibility complex locus on chromosome 19 were identified, and transplantation of hematopoietic stem cells was successfully performed based on immune-matching [80]. Recently, rag ${ }^{\mathrm{E} 450 \mathrm{fs}}$ mutant zebrafish, which have reduced numbers of functional $\mathrm{T}$ and $\mathrm{B}$ cells, were successfully created, and these animals could be used as universal recipients for allograft cell transplantation. This is the first established immunocompromised zebrafish model, and it may produce a new era of stem cell self-renewal and large-scale cell transplantation studies [83]. These advances provide unique opportunities to investigate the mechanisms of engraftment.

\section{Concluding Remarks}

AML is a heterogeneous disorder characterized by acquired genetic changes in hematopoietic progenitor cells. Numerous recurrent gene fusions and point mutations have been identified over the past several decades. However, the mechanism by which these identified alterations interact to induce AML and distinguish driver from passenger mutations in leukemogenesis remains unknown. Chemotherapy is currently used as the standard treatment for AML, except for AML-M3, which shows a good therapeutic response when treated with all-trans retinoic acid (ATRA) or arsenic trioxide, and the majority of AML patients relapse after complete remission or acquire drug resistance, indicating the need for efficacious therapeutic strategies. Several small molecule inhibitors have been developed that showed efficacy in preclinical studies; however, approval of these agents for clinical treatment is still challenging.

The zebrafish genome has been fully sequenced and has a substantial number of conserved genes compared to the human genome. The genetic and cellular levels of hematopoiesis are conserved between these organisms. Several zebrafish models associated with myeloid malignancies have been previously reported, including transgenic zebrafish that exhibit K-RASG12D, NUP98-HOXA9, Stat5 (H298R), Stat5 (N714F), NPM1, tel-jak2a, AML1-ETO, FLt3-ITD, FLT3TKD (D835Y), MYCN, or MOZ/TIF2 expression. Several zebrafish transgenic technologies have also been introduced, including Tol2-mediated transgenesis and GFP-mCherry transgenic lines driven by a myeloid-specific spil promoter. In addition, xenograft zebrafish models used for highthroughput drug screening and leukemia xenotransplantation in zebrafish used for in vivo chemotherapy response assays have also been addressed.

\section{Conflict of Interests}

The authors declare that they have no conflict of interests regarding the publication of this paper.

\section{Acknowledgment}

This study was supported by a research grant from the National Science Council (NSC 100-2320-B-002-074-MY3 and NSC-102-2628-B-002-029-MY3), Taiwan.

\section{References}

[1] S. H. Swerdlow, N. C. Harris, E. S. Jaffe et al., WHO Classification of Tumours of Haematopoietic and Lymphoid Tissues, International Agency of Research on Cancer (IARC), Lyon, France.

[2] Y. Koh, I. Kim, J.-Y. Bae et al., "Prognosis of secondary acute myeloid leukemia is affected by the type of the preceding hematologic disorders and the presence of trisomy 8," Japanese Journal of Clinical Oncology, vol. 40, no. 11, pp. 1037-1045, 2010.

[3] F. Ferrara and C. A. Schiffer, "Acute myeloid leukaemia in adults," The Lancet, vol. 381, no. 9865, pp. 484-495, 2013. 
[4] M. R. O’Donnell, C. N. Abboud, J. Altman et al., "Acute myeloid leukemia," Journal of the National Comprehensive Cancer Network, vol. 10, no. 8, pp. 984-1021, 2012.

[5] S.-J. Chen, Y. Shen, and Z. Chen, "A panoramic view of acute myeloid leukemia," Nature Genetics, vol. 45 , no. 6, pp. 586-587, 2013.

[6] B. Pruvot, A. Jacquel, N. Droin et al., "Leukemic cell xenograft in zebrafish embryo for investigating drug efficacy," Haematologica, vol. 96, no. 4, pp. 612-616, 2011.

[7] Q. Li and J. Uitto, "Zebrafish as a model system to study skin biology and pathology," Journal of Investigative Dermatology, vol. 134, no. 6, article e21, 2014.

[8] S. A. Renshaw and N. S. Trede, "A model 450 million years in the making: zebrafish and vertebrate immunity," Disease Models \& Mechanisms, vol. 5, no. 1, pp. 38-47, 2012.

[9] G. J. Lieschke and N. S. Trede, "Fish immunology," Current Biology, vol. 19, no. 16, pp. R678-R682, 2009.

[10] G. B. Pliss, M. A. Zabezhinski, A. S. Petrov, and V. V. Khudoley, "Peculiarities of N-nitramines carcinogenic action," Archiv für Geschwulstforschung, vol. 52, no. 8, pp. 629-634, 1982.

[11] D. M. Langenau, D. Traver, A. A. Ferrando et al., "Myc-induced T cell leukemia in transgenic zebrafish," Science, vol. 299, no. 5608, pp. 887-890, 2003.

[12] W. Zheng, Z. Li, A. T. Nguyen, C. Li, A. Emelyanov, and Z. Gong, "Xmrk, Kras and Myc transgenic zebrafish liver cancer models share molecular signatures with subsets of human hepatocellular carcinoma," PLoS ONE, vol. 9, no. 3, Article ID e91179, 2014.

[13] M. Schiavone, E. Rampazzo, A. Casari et al., "Zebrafish reporter lines reveal in vivo signaling pathway activities involved in pancreatic cancer," Disease Models \& Mechanisms, vol. 7, no. 7, pp. 883-894, 2014.

[14] X. Le, E. K. Pugach, S. Hettmer et al., "A novel chemical screening strategy in zebrafish identifies common pathways in embryogenesis and rhabdomyosarcoma development," Development, vol. 140, no. 11, pp. 2354-2364, 2013.

[15] D. Traver, B. H. Paw, K. D. Poss, W. T. Penberthy, S. Lin, and L. I. Zon, "Transplantation and in vivo imaging of multilineage engraftment in zebrafish bloodless mutants," Nature Immunology, vol. 4, no. 12, pp. 1238-1246, 2003.

[16] A. J. Davidson and L. I. Zon, "The 'definitive' (and 'primitive') guide to zebrafish hematopoiesis," Oncogene, vol. 23, no. 43, pp. 7233-7246, 2004.

[17] J. L. Galloway and L. I. Zon, “3 ontogeny of hematopoiesis: examining the emergence of hematopoietic cells in the vertebrate embryo," Current Topics in Developmental Biology, vol. 53, pp. 139-158, 2003.

[18] P. Herbomel, B. Thisse, and C. Thisse, "Ontogeny and behaviour of early macrophages in the zebrafish embryo," Development, vol. 126, no. 17, pp. 3735-3745, 1999.

[19] M. O. Crowhurst, J. E. Layton, and G. J. Lieschke, "Developmental biology of zebrafish myeloid cells," The International Journal of Developmental Biology, vol. 46, no. 4, pp. 483-492, 2002.

[20] E. C. Liao, B. H. Paw, A. C. Oates, S. J. Pratt, J. H. Postlethwait, and L. I. Zon, "SCL/Tal-1 transcription factor acts downstream of cloche to specify hematopoietic and vascular progenitors in zebrafish," Genes and Development, vol. 12, no. 5, pp. 621-626, 1998.

[21] M. A. Thompson, D. G. Ransom, S. J. Pratt et al., "The cloche and spadetail genes differentially affect hematopoiesis and vasculogenesis," Developmental Biology, vol. 197, no. 2, pp. 248269, 1998.
[22] S. Sumanas, T. Jorniak, and S. Lin, "Identification of novel vascular endothelial-specific genes by the microarray analysis of the zebrafish cloche mutants," Blood, vol. 106, no. 2, pp. 534541, 2005.

[23] V. N. Pham, N. D. Lawson, J. W. Mugford et al., "Combinatorial function of ETS transcription factors in the developing vasculature," Developmental Biology, vol. 303, no. 2, pp. 772-783, 2007.

[24] M. L. Kalev-Zylinska, J. A. Horsfield, M. V. C. Flores et al., "Runx1 is required for zebrafish blood and vessel development and expression of a human RUNX1-CBF2T1 transgene advances a model for studies of leukemogenesis," Development, vol. 129, no. 8, pp. 2015-2030, 2002.

[25] C. E. Burns, T. DeBlasio, Y. Zhou, J. Zhang, L. Zon, and S. D. Nimer, "Isolation and characterization of runxa and runxb, zebrafish members of the runt family of transcriptional regulators," Experimental Hematology, vol. 30, no. 12, pp. 1381$1389,2002$.

[26] E. Murayama, K. Kissa, A. Zapata et al., "Tracing hematopoietic precursor migration to successive hematopoietic organs during zebrafish development," Immunity, vol. 25, no. 6, pp. 963-975, 2006.

[27] H. Jin, J. Xu, and Z. Wen, "Migratory path of definitive hematopoietic stem/progenitor cells during zebrafish development," Blood, vol. 109, no. 12, pp. 5208-5214, 2007.

[28] H. W. Detrich III, M. W. Kieran, F. Y. Chan et al., "Intraembryonic hematopoietic cell migration during vertebrate development," Proceedings of the National Academy of Sciences of the United States of America, vol. 92, no. 23, pp. 10713-10717, 1995.

[29] L. J. Patterson, M. Gering, and R. Patient, "Scl is required for dorsal aorta as well as blood formation in zebrafish embryos," Blood, vol. 105, no. 9, pp. 3502-3511, 2005.

[30] L. J. Patterson, M. Gering, C. E. Eckfeldt et al., "The transcription factors $\mathrm{Scl}$ and Lmo2 act together during development of the hemangioblast in zebrafish," Blood, vol. 109, no. 6, pp. 23892398, 2007.

[31] H. Zhu, D. Traver, A. J. Davidson et al., "Regulation of the $l m o 2$ promoter during hematopoietic and vascular development in zebrafish," Developmental Biology, vol. 281, no. 2, pp. 256-269, 2005.

[32] K. A. Dooley, A. J. Davidson, and L. I. Zon, "Zebrafish scl functions independently in hematopoietic and endothelial development," Developmental Biology, vol. 277, no. 2, pp. 522536, 2005.

[33] A. B. Cantor and S. H. Orkin, "Transcriptional regulation of erythropoiesis: an affair involving multiple partners," Oncogene, vol. 21, no. 21, pp. 3368-3376, 2002.

[34] J. F. Amatruda and L. I. Zon, "Dissecting hematopoiesis and disease using the zebrafish," Developmental Biology, vol. 216, no. 1, pp. 1-15, 1999.

[35] E. W. Scott, M. C. Simon, J. Anastasi, and H. Singh, "Requirement of transcription factor PU.1 in the development of multiple hematopoietic lineages," Science, vol. 265, no. 5178, pp. 15731577, 1994.

[36] C. M. Bennett, J. P. Kanki, J. Rhodes et al., "Myelopoiesis in the zebrafish, Danio rerio," Blood, vol. 98, no. 3, pp. 643-651, 2001.

[37] E. J. Paik and L. I. Zon, "Hematopoietic development in the zebrafish," International Journal of Developmental Biology, vol. 54, no. 6-7, pp. 1127-1137, 2010.

[38] C. E. Burns, D. Traver, E. Mayhall, J. L. Shepard, and L. I. Zon, "Hematopoietic stem cell fate is established by the Notch-Runx pathway," Genes \& Development, vol. 19, no. 19, pp. 2331-2342, 2005. 
[39] M. Gering and R. Patient, "Hedgehog signaling is required for adult blood stem cell formation in zebrafish embryos," Developmental Cell, vol. 8, no. 3, pp. 389-400, 2005.

[40] G. W. Stuart, J. V. McMurray, and M. Westerfield, "Replication, integration and stable germ-line transmission of foreign sequences injected into early zebrafish embryos," Development, vol. 103, no. 2, pp. 403-412, 1988.

[41] G. W. Stuart, J. R. Vielkind, J. V. McMurray, and M. Westerfield, "Stable lines of transgenic zebrafish exhibit reproducible patterns of transgene expression," Development, vol. 109, no. 3, pp. 577-584, 1990.

[42] P. Culp, C. Nüsslein-Volhard, and N. Hopkins, "High-frequency germ-line transmission of plasmid DNA sequences injected into fertilized zebrafish eggs," Proceedings of the National Academy of Sciences of the United States of America, vol. 88, no. 18, pp. 7953-7957, 1991.

[43] A. Deiters and J. A. Yoder, "Conditional transgene and gene targeting methodologies in zebrafish," Zebrafish, vol. 3, no. 4, pp. 415-429, 2006.

[44] K. Kawakami, A. Shima, and N. Kawakami, "Identification of a functional transposase of the Tol2 element, an Ac-like element from the Japanese medaka fish, and its transposition in the zebrafish germ lineage," Proceedings of the National Academy of Sciences of the United States of America, vol. 97, no. 21, pp. 1140311408, 2000.

[45] A. Urasaki, G. Morvan, and K. Kawakami, "Functional dissection of the Tol 2 transposable element identified the minimal cissequence and a highly repetitive sequence in the subterminal region essential for transposition," Genetics, vol. 174, no. 2, pp. 639-649, 2006.

[46] X. Huang, A. T. Nguyen, Z. Li, A. Emelyanov, S. Parinov, and Z. Gong, "One step forward: the use of transgenic zebrafish tumor model in drug screens," Birth Defects Research Part C: Embryo Today, vol. 93, no. 2, pp. 173-181, 2011.

[47] A. C. Ward, D. O. McPhee, M. M. Condron et al., "The zebrafish spil promoter drives myeloid-specific expression in stable transgenic fish," Blood, vol. 102, no. 9, pp. 3238-3240, 2003.

[48] K. Hsu, D. Traver, J. L. Kutok et al., "The pu.1 promoter drives myeloid gene expression in zebrafish," Blood, vol. 104, no. 5, pp. 1291-1297, 2004.

[49] E. K. Cho, S. M. Bang, J. Y. Ahn et al., "Prognostic value of AML 1/ETO fusion transcripts in patients with acute myelogenous leukemia," The Korean Journal of Internal Medicine, vol. 18, no. 1, pp. 13-20, 2003.

[50] S. Zhao, Y. Zhang, K. Sha et al., "KRAS (G12D) cooperates with AML1/ETO to initiate a mouse model mimicking human acute myeloid Leukemia," Cellular Physiology and Biochemistry, vol. 33, no. 1, pp. 78-87, 2014.

[51] F. J. Giles, A. Keating, A. H. Goldstone, I. Avivi, C. L. Willman, and H. M. Kantarjian, "Acute myeloid leukemia," Hematology/the Education Program of the American Society of Hematology, pp. 73-110, 2002.

[52] G. Ghannam, A. Takeda, T. Camarata, M. A. Moore, A. Viale, and N. R. Yaseen, "The oncogene Nup98-HOXA9 induces gene transcription in myeloid cells," Journal of Biological Chemistry, vol. 279, no. 2, pp. 866-875, 2004.

[53] M. Carapeti, R. C. T. Aguiar, J. M. Goldman, and N. C. P. Cross, "A novel fusion between $\mathrm{MOZ}$ and the nuclear receptor coactivator TIF2 in acute myeloid leukemia," Blood, vol. 91, no. 9, pp. 3127-3133, 1998.
[54] B. J. P. Huntly, H. Shigematsu, K. Deguchi et al., "MOZ-TIF2, but not BCR-ABL, confers properties of leukemic stem cells to committed murine hematopoietic progenitors," Cancer Cell, vol. 6, no. 6, pp. 587-596, 2004.

[55] K. Deguchi, P. M. Ayton, M. Carapeti et al., "MOZ-TIF2induced acute myeloid leukemia requires the MOZ nucleosome binding motif and TIF2-mediated recruitment of CBP," Cancer Cell, vol. 3, no. 3, pp. 259-271, 2003.

[56] S. Xi, Q. Zhang, W. E. Gooding, T. E. Smithgall, and J. R. Grandis, "Constitutive activation of Stat5b contributes to carcinogenesis in vivo," Cancer Research, vol. 63, no. 20, pp. 6763-6771, 2003.

[57] T. S. Lin, S. Mahajan, and D. A. Frank, "STAT signaling in the pathogenesis and treatment of leukemias," Oncogene, vol. 19, no. 21, pp. 2496-2504, 2000.

[58] L.-J. Shen, F.-Y. Chen, Y. Zhang et al., "MYCN transgenic zebrafish model with the characterization of acute myeloid leukemia and altered hematopoiesis," PLoS ONE, vol. 8, no. 3, Article ID e59070, 2013.

[59] H. Kawagoe, A. Kandilci, T. A. Kranenburg, and G. C. Grosveld, "Overexpression of N-Myc rapidly causes acute myeloid leukemia in mice," Cancer Research, vol. 67, no. 22, pp. 10677-10685, 2007.

[60] M. Mallardo, A. Caronno, G. Pruneri et al., "NPMc+ and FLT3ITD mutations cooperate in inducing acute leukaemia in a novel mouse model," Leukemia, vol. 27, no. 11, pp. 2248-2251, 2013.

[61] X. Le, D. M. Langenau, M. D. Keefe, J. L. Kutok, D. S. Neuberg, and L. I. Zon, "Heat shock-inducible Cre/Lox approaches to induce diverse types of tumors and hyperplasia in transgenic zebrafish," Proceedings of the National Academy of Sciences of the United States of America, vol. 104, no. 22, pp. 9410-9415, 2007.

[62] A. M. Forrester, C. Grabher, E. R. Mcbride et al., "NUP98HOXA9-transgenic zebrafish develop a myeloproliferative neoplasm and provide new insight into mechanisms of myeloid leukaemogenesis," British Journal of Haematology, vol. 155, no. 2, pp. 167-181, 2011.

[63] E. Kroon, U. Thorsteinsdottir, N. Mayotte, T. Nakamura, and G. Sauvageau, "NUP98-HOXA9 expression in hemopoietic stem cells induces chronic and acute myeloid leukemias in mice," The EMBO Journal, vol. 20, no. 3, pp. 350-361, 2001.

[64] M. Iwasaki, T. Kuwata, Y. Yamazaki et al., "Identification of cooperative genes for NUP98-HOXA9 in myeloid leukemogenesis using a mouse model," Blood, vol. 105, no. 2, pp. 784-793, 2005.

[65] R. S. Lewis, S. E. M. Stephenson, and A. C. Ward, "Constitutive activation of zebrafish Stat5 expands hematopoietic cell populations in vivo," Experimental Hematology, vol. 34, no. 2, pp. 179$187,2006$.

[66] N. Bolli, E. M. Payne, C. Grabher et al., "Expression of the cytoplasmic NPM1 mutant (NPMc+) causes the expansion of hematopoietic cells in zebrafish," Blood, vol. 115, no. 16, pp. 33293340, 2010.

[67] S. M. N. Onnebo, M. M. Condron, D. O. McPhee, G. J. Lieschke, and A. C. Ward, "Hematopoietic perturbation in zebrafish expressing a tel-jak2a fusion," Experimental Hematology, vol. 33, no. 2, pp. 182-188, 2005.

[68] J. R. J. Yeh, K. M. Munson, Y. L. Chao, Q. P. Peterson, C. A. Macrae, and R. T. Peterson, "AML1-ETO reprograms hematopoietic cell fate by downregulating scl expression," Development, vol. 135, no. 2, pp. 401-410, 2008. 
[69] B.-L. He, X. Shi, C. H. Man et al., "Functions of flt3 in zebrafish hematopoiesis and its relevance to human acute myeloid leukemia," Blood, vol. 123, no. 16, pp. 2518-2529, 2014.

[70] J. Zhuravleva, J. Paggetti, L. Martin et al., "MOZ/TIF2-induced acute myeloid leukaemia in transgenic fish," British Journal of Haematology, vol. 143, no. 3, pp. 378-382, 2008.

[71] J. R. J. Yeh, K. M. Munson, K. E. Elagib, A. N. Goldfarb, D. A. Sweetser, and R. T. Peterson, "Discovering chemical modifiers of oncogene-regulated hematopoietic differentiation," Nature Chemical Biology, vol. 5, no. 4, pp. 236-243, 2009.

[72] P. Goldsmith, "Zebrafish as a pharmacological tool: the how, why and when," Current Opinion in Pharmacology, vol. 4, no. 5, pp. 504-512, 2004.

[73] L. M. J. Lee, E. A. Seftor, G. Bonde, R. A. Cornell, and M. J. C. Hendrix, "The fate of human malignant melanoma cells transplanted into zebrafish embryos: assessment of migration and cell division in the absence of tumor formation," Developmental Dynamics, vol. 233, no. 4, pp. 1560-1570, 2005.

[74] M. Konantz, T. B. Balci, U. F. Hartwig et al., "Zebrafish xenografts as a tool for in vivo studies on human cancer," Annals of the New York Academy of Sciences, vol. 1266, no. 1, pp. 124-137, 2012.

[75] Y. Zhang, J. Wang, J. Wheat et al., "AML1-ETO mediates hematopoietic self-renewal and leukemogenesis through a $\mathrm{COX} / \beta$ catenin signaling pathway.", Blood, vol. 121, no. 24, pp. 49064916, 2013.

[76] J. W. Astin, S. M. F. Jamieson, T. C. Y. Eng et al., "An in vivo anti-lymphatic screen in zebrafish identifies novel inhibitors of mammalian lymphangiogenesis and lymphatic-mediated metastasis," Molecular Cancer Therapeutics, vol. 13, no. 10, pp. 2450-2462, 2014.

[77] D. P. Corkery, G. Dellaire, and J. N. Berman, "Leukaemia xenotransplantation in zebrafish-chemotherapy response assay in vivo," British Journal of Haematology, vol. 153, no. 6, pp. 786789, 2011.

[78] B. Zhang, Y. Shimada, J. Kuroyanagi, N. Umemoto, Y. Nishimura, and T. Tanaka, "Quantitative phenotyping-based in vivo chemical screening in a zebrafish model of leukemia stem cell xenotransplantation," PLoS ONE, vol. 9, no. 1, Article ID e85439, 2014.

[79] C. E. Willett, A. Cortes, A. Zuasti, and A. G. Zapata, "Early hematopoiesis and developing lymphoid organs in the zebrafish," Developmental Dynamics, vol. 214, no. 4, pp. 323-336, 2014.

[80] J. L. O. de Jong, C. E. Burns, A. T. Chen et al., "Characterization of immune-matched hematopoietic transplantation in zebrafish," Blood, vol. 117, no. 16, pp. 4234-4242, 2011.

[81] D. Traver, A. Winzeler, H. M. Stern et al., "Effects of lethal irradiation in zebrafish and rescue by hematopoietic cell transplantation," Blood, vol. 104, no. 5, pp. 1298-1305, 2004.

[82] A. C. H. Smith, A. R. Raimondi, C. D. Salthouse et al., "High-throughput cell transplantation establishes that tumorinitiating cells are abundant in zebrafish T-cell acute lymphoblastic leukemia," Blood, vol. 115, no. 16, pp. 3296-3303, 2010.

[83] Q. Tang, N. S. Abdelfattah, J. S. Blackburn et al., "Optimized cell transplantation using adult rag2 mutant zebrafish," Nature Methods, vol. 11, no. 8, pp. 821-824, 2014. 


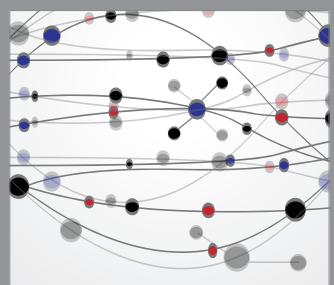

The Scientific World Journal
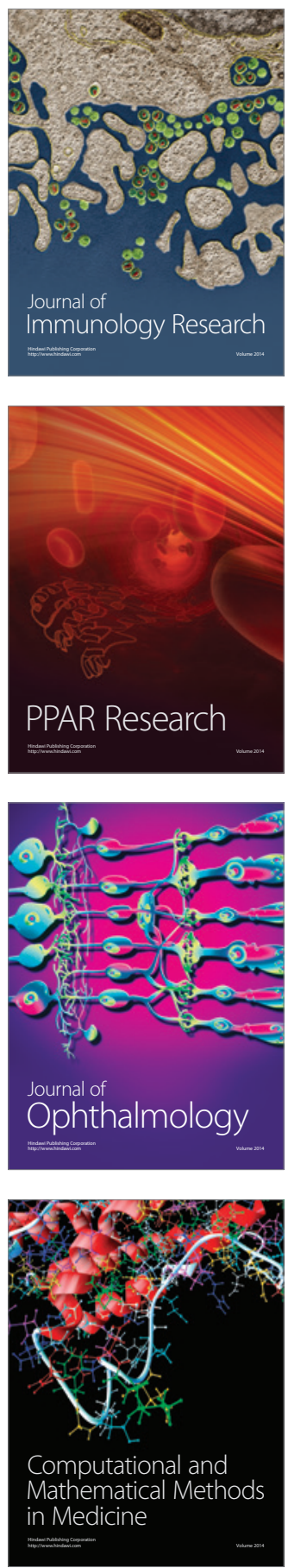

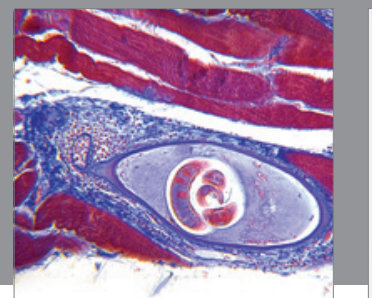

Gastroenterology

Research and Practice
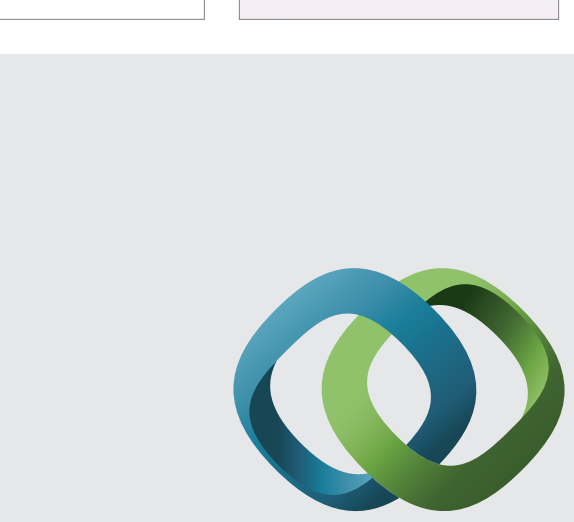

\section{Hindawi}

Submit your manuscripts at

http://www.hindawi.com
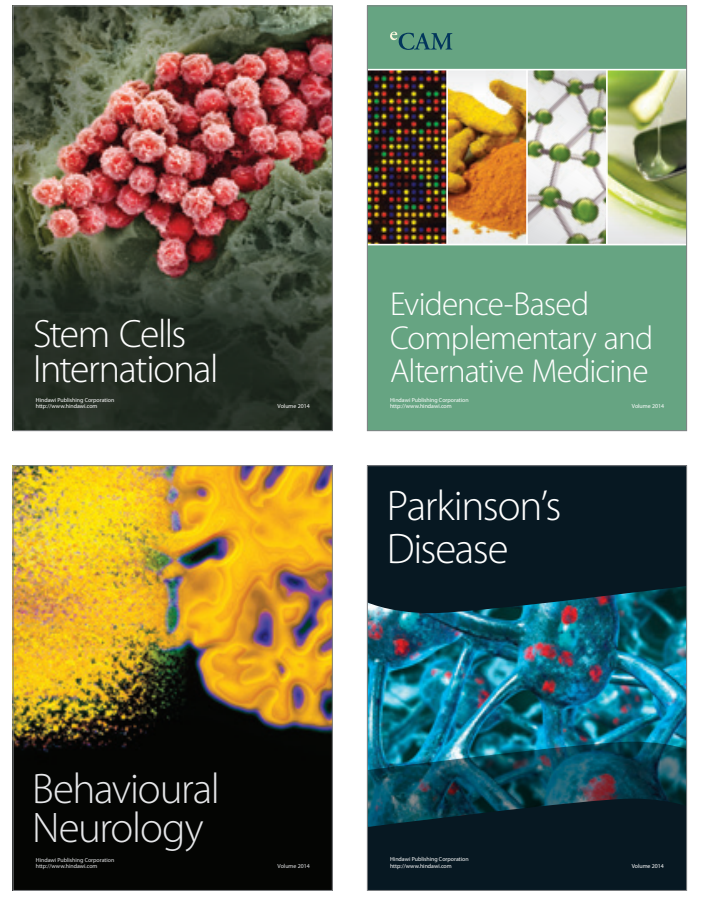
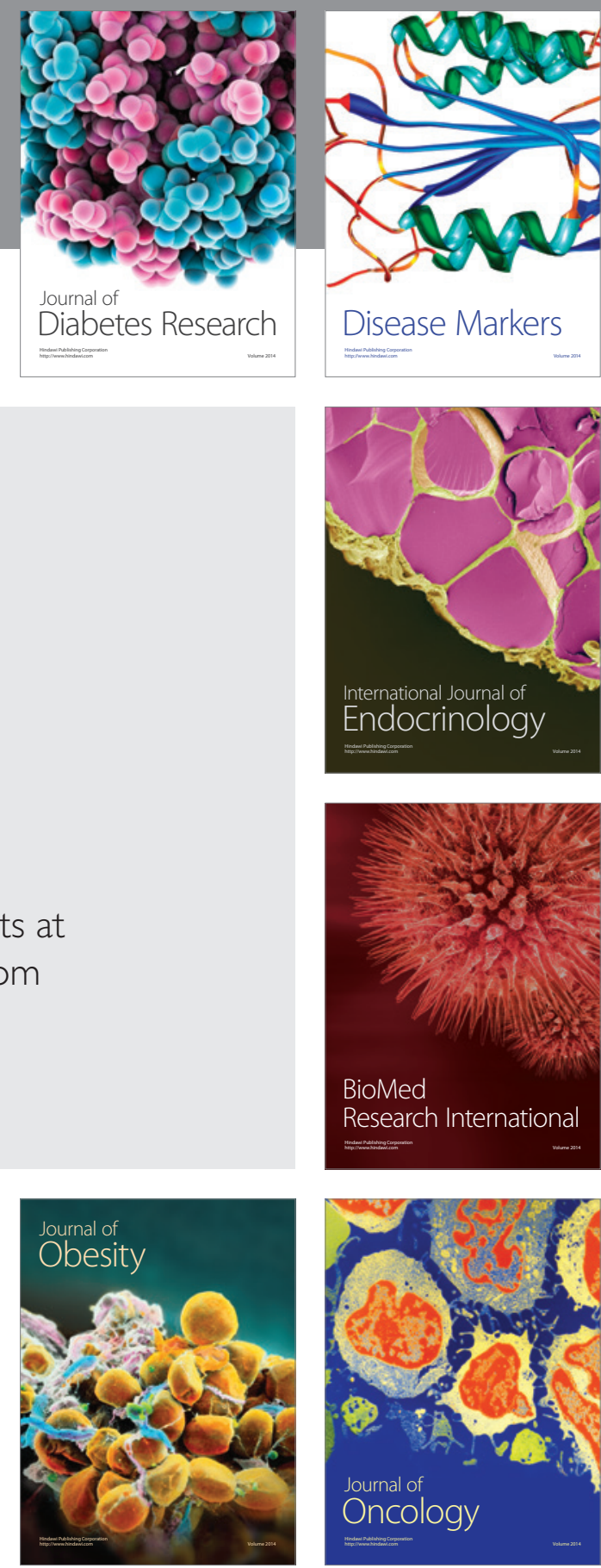

Disease Markers
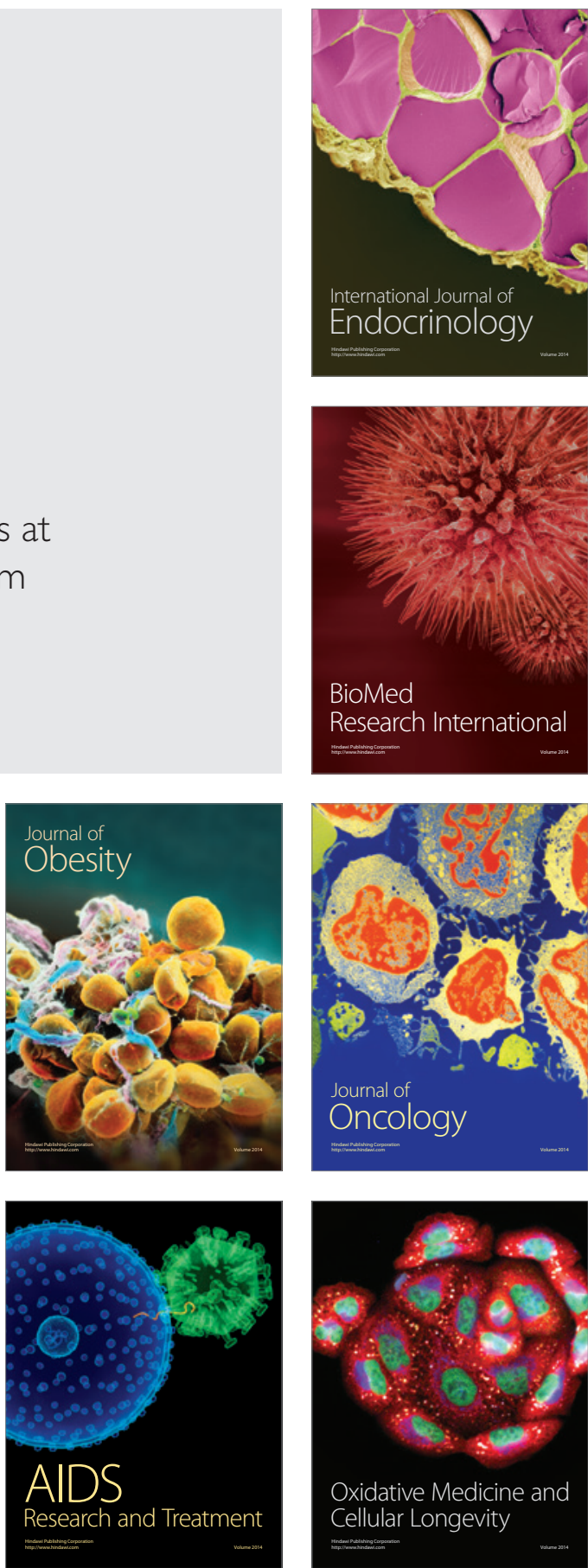